Florida International University FIU Digital Commons

$10-10-2012$

\title{
Can Intersensory Redundancy and Social Contingency Enhance Memory in Bobwhite Quail Hatchlings?
}

Namitha Raju

Florida International University, namitha@gmail.com

DOI: $10.25148 /$ etd.FI13040106

Follow this and additional works at: https://digitalcommons.fiu.edu/etd

\section{Recommended Citation}

Raju, Namitha, "Can Intersensory Redundancy and Social Contingency Enhance Memory in Bobwhite Quail Hatchlings?" (2012). FIU Electronic Theses and Dissertations. 811.

https://digitalcommons.fiu.edu/etd/811 


\title{
FLORIDA INTERNATIONAL UNIVERSITY
}

Miami, Florida

\section{CAN INTERSENSORY REDUNDANCY AND SOCIAL CONTINGENCY ENHANCE MEMORY IN BOBWHITE QUAIL HATCHLINGS?}

\author{
A thesis submitted in partial fulfillment of \\ the requirements for the degree of \\ MASTER OF SCIENCE \\ in \\ PSYCHOLOGY
}

by

Namitha Raju 
To: Dean Kenneth G. Furton

College of Arts and Sciences

This thesis, written by Namitha Raju, and entitled Can Intersensory Redundancy and Social Contingency Enhance Memory in Bobwhite Quail Hatchlings?, having been approved in respect to style and intellectual content, is referred to you for judgment.

We have read this thesis and recommend that it be approved.

$\begin{array}{r}\hline \text { Mary Levitt } \\ \hline \text { Lorriane Bahrick } \\ \hline \text { Robert Lickliter, Major Professor }\end{array}$

Date of Defense: October 10, 2012

The thesis of Namitha Raju is approved.

\begin{tabular}{r} 
Dean Kenneth G. Furton \\
College of Arts and Sciences \\
\hline Dean Lakshmi N. Reddi \\
University Graduate School
\end{tabular}

Florida International University, 2013 


\section{ACKNOWLEDGMENTS}

I am sincerely grateful to my major professor, Dr. Robert Lickliter who has provided invaluable guidance and support throughout this project. I would also like to thank my committee member, Dr. Lorraine Bahrick, for her constructive feedback on this project. Moreover, I am very grateful to our lab manager and the undergraduate research assistants who have helped me in collecting data. Lastly, I would like to acknowledge the continual encouragement of my husband without whose support I would not have been exposed to this wonderful world of scientific research. 


\title{
ABSTRACT OF THE THESIS \\ CAN INTERSENSORY REDUNDANCY AND SOCIAL CONTINGENCY ENHANCE MEMORY IN BOBWHITE QUAIL HATCHLINGS?
}

\author{
by \\ Namitha Raju \\ Florida International University, 2012 \\ Miami, Florida \\ Professor Robert Lickliter, Major Professor
}

Recent findings indicate that bimodal-redundant stimulation promotes perceptual learning and recruits attention to amodal properties in non-human as well as human infants. However it is not clear if bimodal-redundant stimulation can also facilitate memory during the postnatal period. Moreover, most animal and human studies have employed an operant paradigm to study memory, but have not compared the effectiveness of contingent versus passive presentation of information on memory. The current study investigated the role of unimodal versus bimodal presentation and, the role of a contingent versus passive exposure in memory retention in the bobwhite quail (Colinus virginianus). Results revealed that contingently trained chicks demonstrated a preference for the familiarized call under both unimodal and bimodal conditions. Between-group analyses revealed that the contingent-bimodal group preferred the familiarized call as compared to the passive-bimodal group. These results indicate that the contingency paradigm accompanied with the bimodal stimulus type facilitated memory during early development. 


\section{TABLE OF CONTENTS}

CHAPTER $\quad$ PAGE

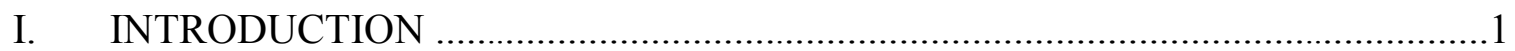

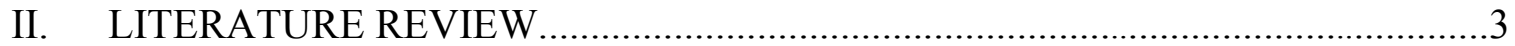

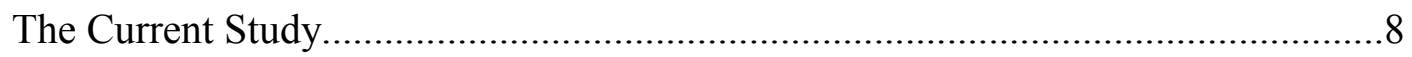

Research Questions and Hypotheses....................................................................10

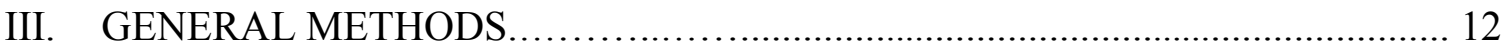

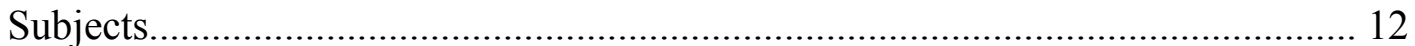

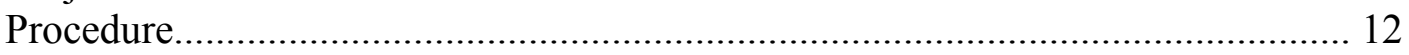

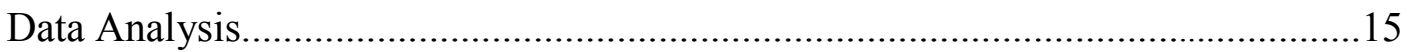

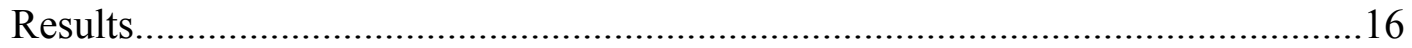

IV. DISCUSSION

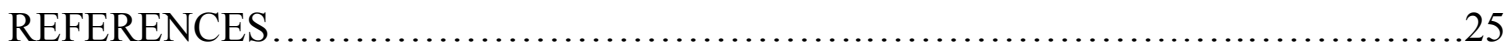




\section{CHAPTER I.}

\section{Introduction}

There is a large body of research in the human and non-human animal literature that has focused on the dynamics of early memory development. The study of early memory development has come a long way from its initial concerns with whether and how early infants learn, to its current focus on how long infants can remember what they learned and under what conditions (Rovee-Collier, 1996). Researchers have found that infant memory is influenced by several factors, including amount and distribution of training (Ohr, Fagen, Rovee-Collier, Hayne, \& Linde, 1989), the match between the focal object of learning and contextual cues present at acquisition and recall (Rovee-Collier, Schechter, Shyi, \& Shields, 1992), the amount of information in terms of number of components in a sequence (Bauer, 1995), postevent information (if novel or familiar) along with its location (whether central or peripheral to the target) and timing (Boller, Grabelle, \& Rovee-Collier, 1995), the organization of information to be retained (i.e., when ordered causally or arbitrarily, see Bauer, 1997), and reminder techniques such as reactivation and reinstatement (Rovee-Collier, 1995), to name a few. While these findings from memory research have informed us about a number of mechanisms involved in learning and memory retrieval during early development, less attention has been paid to understanding infant memory by manipulating the perceptual experience of young organisms.

A study by Bahrick, Gogate, and Ruiz (2002) illustrates how stimulus properties and contexts can influence memory functioning during early development. Bahrick et al. (2002) found that $5 \frac{1}{2}$ month-old infants showed greater discrimination and memory for 
dynamic actions (e.g., a lady combing hair, or brushing teeth, or blowing bubbles) than the faces of the actors when tested seven weeks after initial exposure. In this study, infants remembered the repetitive dynamic actions, but not the faces of the actors performing the action. When these infants were tested in static contexts, faces were remembered as compared to actions, but only for a short amount of time. These results imply that infants pay attention to different stimulus properties in static versus dynamic contexts and that dynamic information is retained longer than static information. The findings from Bahrick et al.'s (2002) study also provide motivation to explore other aspects of stimulation that may aid or interfere with memory retention. 


\section{CHAPTER II.}

\section{Literature Review}

More than two decades of research has demonstrated that young infants are capable of intersensory perception and can detect, learn, and remember amodal information during early development (e.g., Bahrick \& Pickens, 1994; Gibson \& Pick, 2000; Lewkowicz, 2000; Lewkowicz \& Lickliter, 1994; Meltzoff \& Kuhl, 1994). Amodal information is information that is common across two or more sense modalities, such as synchrony, rhythm, tempo, duration, and intensity. For example, the lip movement and sounds of a person speaking provide intersensory redundancy, since the information is temporally synchronous, spatially collocated, and conveys the same information across visual and auditory modalities. Young perceivers have been shown to selectively attend to amodal information at the expense of unrelated sights and sounds present in the flux of concurrent stimulation in their environment (Bahrick \& Pickens, 1994). Given that selective attention is fundamental to what is perceived and learned, identifying the processes that recruit selective attention is key to understanding the course of learning and memory during early development.

Bahrick and Lickliter $(2000,2002)$ proposed the 'Intersensory Redundancy Hypothesis' (IRH), which addresses how selective attention is allocated to different properties of events to guide perceptual learning during early development. The IRH makes four predictions that address how properties of stimulation might be processed in unimodal and multimodal stimulation: 1) The first prediction is termed as intersensory facilitation which suggests that redundant multimodal stimulation selectively guides attention to the amodal properties of events to a greater extent than unimodal stimulation. 
2) The second prediction of IRH is called unimodal facilitation which states that in nonredundant unimodal stimulation, modality-specific properties are more salient than are the same properties in bimodal synchronous stimulation. 3) According to the third prediction, as attention becomes more efficient and flexible with experience, detection of both amodal and modality specific properties should be evident in redundant, bimodal stimulation as well as in nonredundant, unimodal stimulation. 4) The fourth prediction of IRH states that while intersensory facilitation and unimodal facilitation are more evident during early development, they are also applicable whenever task difficulty is high compared to one's level of expertise and hence should be apparent across the life-span when task demands are high.

Several studies have supported the predictions of the IRH, establishing it as a useful framework for advancing our understanding of early perceptual and cognitive development (Bahrick \& Lickliter, 2000; Bahrick, Lickliter, Castellanos, \& VaillantMolina, 2010; Flom \& Bahrick, 2007; Lickliter, Bahrick, \& Honeycutt, 2002; Vaillant, Bahrick, \& Lickliter, 2009). The key role of intersensory facilitation has been documented in affect discrimination, rhythm and tempo discrimination, numerical discrimination, abstract rule learning, word comprehension and segmentation, and memory enhancement (Bahrick, Lickliter, Castellanos, \& Vaillant-Molina, 2010; Flom \& Bahrick, 2007; Frank, Slemmer, Marcus, \& Johnson, 2009; Gogate \& Bahrick, 2001; Hollich, Newman, \& Jusczyk, 2005; Jordon, Suanda \& Brannon, 2008; Lickliter, Bahrick, \& Honeycutt, 2004). Recognizing the role of intersensory facilitation in early perceptual and cognitive development has opened up new avenues for exploring the links between attention, perceptual processing and memory processes. 
For example, Lickliter, Bahrick, \& Honeycutt, (2004) demonstrated that intersensory facilitation not only recruits selective attention for amodal properties but also enhances memory for these same properties in prenatal development. Bobwhite quail chicks that received redundant bimodal (audiovisual) exposure to a specific variant of a bobwhite maternal call in a bimodal redundant fashion for 10 minutes every hour for the last 24 hours prior to hatching preferred the maternal call 48 hours after hatching, whereas chicks that had received only unimodal auditory exposure failed to prefer the familiarized call by 48 hours after hatch. Moreover, when a brief refamiliarization procedure was provided either at 48 or 72 hours, chicks from the bimodal redundant group continued to prefer the familiar maternal call at 72 and 96 hours following hatching. However, this refamiliarization procedure was not successful with the unimodal auditory group, who again failed to prefer the familiar maternal call at both 72 and 96 hours. The findings from Lickliter et. al's study (2004) show that the factors that aid selective attention and the perception of stimulus properties also enhance memory for those stimulus properties during perinatal development. Since the postnatal environment differs from the more sequestered prenatal environment, it is not clear if the above findings of the facilitative effects of intersensory redundancy during the prenatal period extend to early postnatal development.

Findings from research on the Intersensory Redundancy Hypothesis highlight the importance of redundant bimodal stimulation in recruiting selective attention, that in turn could support or facilitate memory functions. For example, Bahrick and Pickens (1995) found that 3-month olds show memory for object motion (either one large yellow-striped metal washer or a cluster of several small orange metal nuts moving in a horizontal or 
circular path with their natural sounds, providing intersensory redundancy) for as long as three months. These findings were evaluated within the framework of the four-phase attention function that hypothesized that attention to novel and familiar stimuli interact with retention time. The four-phase attention function was characterized by 1) a novelty preference for recent memories, 2) no preference for intermediate memories, 3) familiarity preference for remote memories, and 4) no preference for inaccessible memories. However, Bahrick and Picken's (1995) study focused on documenting the four-phase attention function and did not specifically compare the bimodal-redundant presentation of stimuli with unimodal presentation.

In a related study, Flom and Bahrick (2010) compared the effects of bimodal audiovisual and unimodal visual stimulation (moving toy hammer) on infant's memory. Flom and Bahrick (2012) demonstrated that memory for non-redundant aspects of stimulation (such as orientation of the toy hammer) is a result of selective attention to those aspects of stimulation and is facilitated in unimodal stimulation. However the focus of this study did not include memory for redundant bimodal stimulation. No study has systematically compared the effects of unimodal versus redundant bimodal presentation on long-term memory during postnatal development, even though bimodal redundant stimulation is abundant in real life settings. Comparing the effects of unimodal and bimodal redundant stimulation on memory can help to better understand the role of intersensory redundancy in supporting memory development.

Another neglected topic in the memory development literature is a systematic comparison between the various methods of stimulus presentation. Most studies typically choose one method of presentation, for example, either a contingency paradigm or a 
passive, non-contingent paradigm. In the contingency paradigm, the stimuli is presented contingent on the response of the organism, whereas in a non-contingent paradigm, stimuli is presented passively to the organism, usually for longer periods of time (for example, repeated every hour for several hours or days, see Lickliter \& Hellwell, 1992; Gottlieb, 1993, for examples from animal studies).

Previous studies with precocial birds have demonstrated that a 5-minute contingent exposure to a specific variant of a bobwhite maternal call is sufficient for bobwhite quail chicks to prefer that familiarized call over a novel maternal call after a 24hour delay (Harshaw \& Lickliter, 2007). Other studies using a passive, non-contingent method have required 240 minutes of exposure to the maternal call for chicks to show a preference for the familiarized call in subsequent testing (Foushee \& Lickliter, 2002; Lickliter \& Hellwell, 1992). Moreover, Harshaw, Tougerman, and Lickliter (2008) demonstrated that presenting 1-day old bobwhite hatchlings with a Japanese quail maternal call contingent on their own vocalizations (unimodal auditory presentation) for 5 minutes on a variable-ratio schedule (VR2) resulted in significant preference for the Japanese quail call over the species-specific bobwhite maternal call. The contingency training was thus able to shift the bobwhite neonate's species-typical auditory preference. However it is not known whether the ability to remember familiarized stimuli beyond 24 hours can be enhanced by contingent presentations. Since prior research has shown that reminders of the original stimuli can strengthen the memory trace and help maintain it for longer durations (Campbell \& Jaynes, 1966; Spear, 1973; Rovee-Collier, 1995), it is unclear if a passive methodology is better for memory retention over longer periods as compared to the briefer contingent-training method. 
Research with songbirds has compared different methods of stimuli presentation on learning and memory. For example, studies have assessed the effects of learning from a passive tape playback, a live tutor (birds as well as humans), a socially interactive tutor, as well as computer simulated virtual tutors (Beecher \& Burt, 2004). While these studies have identified social interaction as a key component in facilitating the learning process in altricial songbirds, the focus of these experiments has primarily been to identify the human correlates of human speech learning. These studies have not directly focused on evaluating memory retention as a function of these different types of stimulus presentations.

The goal of the present study was to contribute to the existing memory development literature by exploring how the presentation of stimuli affects memory, by using both contingent and non-contingent passive presentation methods. Further, I explored the effectiveness of redundant versus non-redundant training on memory retention during early postnatal development.

\section{The Current Study}

This study compares the effects of postnatal presentation of unimodal (nonredundant) auditory stimulation and redundant bimodal (audiovisual) stimulation on memory in bobwhite quail using contingent, non-contingent and passive presentation methods. Bobwhite quail chicks were used as subjects because their sensory systems are functional at hatching and they are mobile immediately after hatching, which allows them to be used in behavioral tests (i.e., moving to a preferred location) in the days following hatching. Further, results from previous studies of early perceptual learning with human infants have been replicated using bobwhite quail (Lickliter, Bahrick, \& Honeycutt, 2002; 
Lickliter, Bahrick, \& Honeycutt, 2004; Vaillant, Bahrick, \& Lickliter, 2009). Bahrick and Lickliter (2000) found that 5-month-old infants can discriminate complex rhythmic patterns when presented audiovisually but not when the same information is presented unimodally. Infants who heard and viewed a video of a hammer tapping a rhythm were able to detect changes in the rhythm but were not able to do so when they only heard the tapping or saw the tapping. These findings of intersensory facilitation were replicated by Lickliter, Bahrick, and Honeycutt (2002), where bobwhite embryos were presented either with a variant of the bobwhite maternal call or the call accompanied by a pulsing light that flashed in synchrony with the notes of the maternal call for 10 minutes every hour, for an exposure duration of either 6,12 , or 24 hours. The hatchlings were tested at 24 hours after hatch to determine if they would show a preference for the familiar call over a novel variant of the maternal call. Results showed that the chicks that had received bimodal redundant presentation of the maternal call were able to prefer the familiar call at all exposure durations $(6,12$, and 24 hours) whereas the chicks receiving the unimodal presentation of the call preferred the familiar call only at the longest exposure duration (24 hours).

In the current study bobwhite quail chicks were presented with a specific variant of bobwhite maternal call either 1) unimodally (auditory) or 2) bimodally (redundant audiovisual). Chicks received this exposure either 1) using a contingent presentation method where the stimuli was presented contingent on their own vocalization (FR1 schedule) in a 5-minute training session at 24 hours of age, or 2) using a passive method where the chicks were presented the maternal call for 10 minutes each hour for 24 hours. To determine the effectiveness of the contingency training, an additional non-contingent 
group received the same amount of training (vocalizations) under the same conditions as the contingent group, except that the maternal call that was presented was not contingent on the chick's vocalization. All chicks were tested individually 2 days later at 72 hours of age between the familiar bobwhite maternal call versus a novel variant of the maternal call. A 48-hour delay was chosen since previous postnatal studies have not evaluated memory beyond 24 hours following initial exposure, and this two-day window likely serves as a long-term delay in this rapidly developing precocial avian species.

\section{Research Questions and Hypotheses}

Previous studies have shown the facilitative effect of intersensory redundancy on early perceptual learning in bobwhite quail embryos. While it has been shown that embryos require 240 minutes of unimodal auditory presentation distributed over 24 hours to demonstrate a preference for the familiar maternal call postnatally, only 60 minutes of redundant bimodal (audiovisual) exposure distributed over 6 hours was sufficient for chicks to demonstrate a preference for the familiar maternal call in postnatal testing (Lickliter, Bahrick, \& Honeycutt, 2002). The facilitative effects of intersensory redundancy were found in the domain of memory as well, as bobwhite embryos exposed to audiovisual redundancy demonstrated a significant preference for the familiar call four times longer than when the call was presented unimodally (Lickliter, Bahrick, \& Honeycutt, 2004). The findings raise the question of whether exposure to intersensory redundancy can also facilitate memory in postnatal development.

Further, in previous studies chicks have shown a preference for the familiar call presented unimodally for as long as 24 hours following exposure using contingent as well as passive methods (Lickliter \& Hellwell, 1992; Harshaw \& Lickliter, 2007). It is not 
clear if intersensory redundancy (presented contingently or non-contingently) can enhance memory and in turn the preference for the familiar call for longer than 24 hours after the initial training session.

I hypothesized that: 1) chicks that receive audio-visual redundant training will remember the familiar call at 72 hours of age, given that benefits of intersensory redundancy on prenatal memory retention have been shown in previous studies, 2) chicks presented with the unimodal auditory training will not remember the familiar call at 72 hours of age following hatching. These hypothesis do not make predictions about the effects of training type (contingent versus passive), as there is not enough evidence from previous studies about the effect of contingent versus passive paradigms on memory retention during early development to make a clear prediction. 


\section{CHAPTER III.}

\section{General Methods}

\section{Subjects}

Subjects were 280 incubator-reared bobwhite quail (Colinus virginianus) chicks. Fertile unincubated eggs were received weekly from a commercial supplier and incubated in an incubator maintained at $37.5{ }^{\circ} \mathrm{C}$ and $65-70 \%$ relative humidity. The embryo's age was calculated such that the first day of incubation was Day 0. The possible influence of between-batch variation in behavior was controlled by drawing subjects for each experimental group from three or more different batches (i.e., weeks) of eggs. Following hatching, 15-20 chicks were reared together in standard plastic tubs in a rearing room where the ambient air temperature was maintained at approximately 35C. Chicks had constant access to food and water, except during training and testing sessions.

\section{Procedure}

\section{Contingency Training}

Chicks were trained individually within a large circular arena measuring $130 \mathrm{~cm}$ in diameter and $60 \mathrm{~cm}$ in height. The arena surface was painted white, and a white curtain covered the walls of the arena. A video camera mounted directly above the arena was used for observing the chicks and logging training data. The speakers were mounted to the arena wall and were hidden behind the white curtain. Contingency training took place at 24 hours of age. Bobwhite quail chicks were divided into 2 experimental groups: 1) a unimodal auditory group and 2) a bimodal audio-visual group. Chicks from the unimodal group heard a specific variant of the bobwhite maternal call contingent on their 
own vocalization from either of the speakers in the arena, whereas chicks from the bimodal audio-visual group were exposed to the contingent call paired with a light that flashed in synchrony with the notes of the call. The light was placed on the floor of the arena in a standing position next to the speaker. It was covered with a transparent plastic cup. At the beginning of the training session, if a chick was non-vocal, the maternal call was played for a maximum of 5 times non-contingently to engender a response. The training session lasted 5 minutes.

Non-contingency Training

The training protocol was identical to the contingency training described above except that the maternal call was not played contingent on the chick's vocalizations. Rather, the average number of vocalizations was calculated from the contingent training sessions (thirty). A program was devised using Microsoft Excel to play the thirty vocalizations at specific intervals within the 300 -second trial period (for example, the maternal call was repeated at 4, 7, 14, 19, 26 seconds, and so on). These times were taken from a contingency training session where the chick was actively vocalizing throughout the session. All the chicks in the non-contingent group were presented the maternal calls from the same standardized program. It is possible that a few vocalizations that were played were contingent on the chick's vocalizations. To control for this, the experimenter made sure that if the automated vocalizations were accidently contingent on the chick's vocalizations more than five times during the training trial, the chick was not included in the condition. 


\section{Passive, Non-contingency Training}

Passive training was conducted in a sound attenuated room where 15-20 chicks were housed in a rearing tub from the time they hatched until they were tested. Bobwhite quail chicks were divided into 2 experimental groups as mentioned above. The unimodal auditory group heard a specific variant of the bobwhite maternal call from a speaker that was placed next to the rearing tub. The bimodal audio-visual group received the same maternal call paired with pulsed light that was synchronized to the notes of the call. The audio as well as the audio-visual presentations was delivered from a computer with a javascript program that played the maternal call 10 minutes each hour, for a total of 24 hours (total exposure time $=240$ minutes). Each vocalization was 3 seconds long and there was a 1 second pause between 2 vocalizations. The chicks in the passive group thus heard a total of 3600 vocalizations across the 24 -hour period.

Two variants of the bobwhite maternal call (hereafter referred to as Call A and Call B) served as the familiar and novel call during testing, and were counterbalanced during training. Half of the subjects from each experimental group were trained with Call A and the other half were trained with Call B.

Testing took place 48 hours after training when the chicks were 72 hours of age. The trained chicks were tested individually in a 5 minute simultaneous choice test between the familiar maternal call versus a novel bobwhite maternal call, each of which was played from one of the speakers in the arena's approach areas. Each chick was tested only once. The position of the two maternal calls was counterbalanced to avoid any side bias. Both these maternal calls were played simultaneously and preference for 
the familiar call was determined by recording how much time the chick spent in each of these approach areas during the testing trial.

Testing was conducted for all chicks in the same arena where the contingency training occurred. Two semi-circular approach areas were demarcated around the speakers. These approach areas comprise $5 \%$ of the total arena. At the beginning of the testing session, a chick was placed in the arena equidistant from both the approach areas. All chicks were tested between the familiar version of the maternal call versus a novel maternal call. The sound intensity of each call was adjusted to a maximum peak of $65 \mathrm{db}$ and it was measured from a point that was equidistant to both speakers.

\section{Data Analysis}

The dependent variable of interest was the measure of preference for the familiar maternal call during the testing session. The measures of preference used were 1) the total duration of time spent within the familiar versus the novel approach area, and 2) the total number of entries into the familiar approach area versus the novel approach area. Chicks that failed to spend at least 30 seconds in one approach area were scored as nonresponders. The number of entries into the familiar area was converted into proportion of total entries (PTE) by dividing the number of entries into the familiar area by the number of entries into the familiar and novel area. Duration scores were converted into proportion of total duration (PTD) by dividing the duration of time spent in the familiar area by the total duration spent in the familiar and novel area. Four scenarios were examined by looking at a combination of PTE and PTD: 1) a statistically significant PTD over $50 \%$ and a statistically significant PTE over 50\% would mean that chicks that spent 
a large duration of time in the familiar area and visited the familiar area very often, 2) a statistically significant PTD over 50\% and a non-statistically significant PTE would mean that chicks that spent a large duration of time in the familiar area stayed in the familiar area for longer durations and did not move frequently between the two areas, 3) a nonstatistically significant PTD and a statistically significant PTE over 50\% would mean that the chicks moved to the familiar approach area very often but did not stay there for long, and 4) a non-statistically significant PTD and a non-statistically significant PTE would mean that chicks spent relatively little time in the familiar approach area and did not visit the area often.

The PTD and PTE scores within groups were evaluated using the one-sample $t$ test with an established level of 0.5 . Tests of normality were conducted using the Shapiro-Wilk test. Between-group comparisons included comparing the different stimulus types (unimodal, bimodal), comparing the different stimulus presentations (passive, contingent, and non-contingent), and comparing interactions between stimulus types and stimulus presentations for both PTD and PTE. These were conducted using the non-parametric Mann-Whitney $U$ test and Kruskal-Wallis $\mathrm{H}$ test since the tests of normality indicated that the data was not normally distributed.

\section{Results}

Results from the one sample $t$-test are shown in Table 1. The PTD for the within group comparison test was significantly higher than $50 \%$ for the contingent-bimodal group and the contingent-unimodal group, indicating a preference for the familiarized call. In contrast, the passive-bimodal group, the passive-unimodal group, the 
noncontingent-bimodal group, and the noncontingent-unimodal group did not show a significant preference for either the familiar or the novel call during testing.

Table 1. PTD scores across exposure conditions.

\begin{tabular}{|c|c|c|c|c|c|}
\hline Condition & Mean & SD & $t$ value & $p$ value & $D$ \\
\hline Contingent Bimodal & .65 & .36 & $t(39)=2.71$ & $\mathbf{. 0 1 *}$ & .41 \\
\hline Contingent Unimodal & .60 & .25 & $t(33)=2.32$ & $\mathbf{. 0 2 *}$ & .40 \\
\hline Passive Bimodal & .51 & .29 & $t(40)=0.29$ & .77 & .03 \\
\hline Passive Unimodal & .57 & .30 & $t(39)=1.53$ & .13 & .23 \\
\hline Non-Contingent Bimodal & .58 & .28 & $t(20)=1.34$ & .19 & .28 \\
\hline Non-Contingent Unimodal & .54 & .30 & $t(32)=0.87$ & .39 & .13 \\
\hline
\end{tabular}

The PTE results of the contingent bimodal and the contingent unimodal groups indicate that the chicks in both these conditions frequently visited the familiar area. Additionally the PTE was statistically significant for the passive unimodal group, but the non-significant PTD indicates that even though the chicks frequently visited the familiar area, they did not spend enough time in the familiar area to indicate a preference for the familiar call.

Table 2. PTE scores across exposure conditions.

\begin{tabular}{|c|c|c|c|c|c|}
\hline Condition & Mean & SD & $t$ value & $p$ value & $D$ \\
\hline Contingent Bimodal & .69 & .32 & $t(39)=3.83$ & $\mathbf{. 0 0 *}$ & .87 \\
\hline Contingent Unimodal & .58 & .27 & $t(33)=2.23$ & $\mathbf{. 0 3 *}$ & 1.27 \\
\hline Passive Bimodal & .51 & .26 & $t(40)=0.29$ & .78 & .30 \\
\hline Passive Unimodal & .58 & .22 & $t(39)=1.53$ & $\mathbf{. 0 4 *}$ & 1.69 \\
\hline Non-Contingent Bimodal & .56 & .24 & $t(20)=1.34$ & .23 & 1.52 \\
\hline Non-Contingent Unimodal & .57 & .27 & $t(32)=0.87$ & .11 & .25 \\
\hline
\end{tabular}


The Shapiro-Wilk's test of normality indicated that the PTD's of passiveunimodal $(p=.02)$, contingent-bimodal $(p<.001)$, and noncontingent-unimodal $(p=$ 0.01) groups were not normally distributed. Hence non-parametric tests were used to evaluate between-group preferences. A Mann-Whitney $U$ test revealed no significant difference in preference for the familiar call as a result of the effects of bimodal versus unimodal presentation $(U=5256, \mathrm{z}=-0.46, p=.64, r=.03)$. There was also no main effect of PTE for stimulus type $(U=5315, \mathrm{z}=-0.32, p=.74, r=.02)$. Moreover, a Kruskal-Wallis test revealed no significant difference in memory retention as a result of stimulus presentation (Gp 1, $n=81$ : passive, Gp 2, $n=74$ : contingent, Gp 3, $n=54$ : noncontingent), $\chi^{2}(2, n=209)=4.00, p=.13$. However, a marginal main effect of stimulus presentation was found for the PTE, $\left.\chi^{2}(2, n=209)=5.86, p=.053\right)$ which suggests that the number of times the chicks entered the familiar area differed across different stimulus presentation types.

Interactions between stimulus type and stimulus presentations for PTD were evaluated using the Mann-Whitney $U$ test. Results revealed a significant difference only between the passive-bimodal and the contingent-bimodal group for PTD $(U=598, \mathrm{z}=$ 2.10, $p=.03, r=.23)$. Between-group comparisons of PTE also revealed a significant difference between the passive-bimodal and the contingent-bimodal group $(U=503, \mathrm{z}=$ $-3, p=.00, r=.33)$. Additionally two other group comparisons were statistically significant using the proportion of total entries; these were the contingent-bimodal versus the non contingent-bimodal groups $(U=282, \mathrm{z}=-2.10, p=.03, r=.26)$ and the 
contingent-unimodal versus the contingent-bimodal groups $(U=482, \mathrm{z}=-2.15, p=.03, r$ $=.24)$.

To assess which group had the most number of chicks that exhibited a preference for the familiar call, a measurement of the percentage of chicks in each condition with a PTD score greater than .90 was calculated. The contingent-bimodal group had $45 \%$ of the chicks demonstrating the PTD score above .90 , followed by non-contingent unimodal (24\%), contingent-unimodal (15\%), passive-bimodal (15\%), non-contingent bimodal $(14 \%)$, and passive-unimodal (12\%) groups. 


\section{CHAPTER IV.}

\section{Discussion}

During early development, the selective allocation of attention is critical since the external world is rich in stimulation and the young organism has to attend to certain information while ignoring others. To understand how learning takes place, how memories are formed, or how long information can be remembered during early development, it is important to explore the processes that guide selective attention and perceptual learning. Specifically, the aim of this study was to determine if 1) intersensory facilitation can enhance memory in early postnatal development and if 2) type of stimulus presentation influences learning and memory.

The first goal explored the first prediction of the Intersensory Redundancy Hypothesis in the domain of memory. According to the first prediction, perceptual processing and learning of amodal properties is better facilitated in multimodal stimulation as compared to unimodal stimulation. Numerous studies have shown that bimodal redundant stimulation has facilitated learning in human as well as non-human animal infants. However the role of intersensory redundancy in the domain of memory retention had not been well explored. The current study hypothesized that bimodal redundant stimulation would also enhance memory retention compared to unimodal stimulation in the early postnatal period. Results showed a lack of main effect of stimulus type (unimodal versus bimodal). Even though chicks in the contingent-bimodal group demonstrate a greater mean PTD (67\%) compared to the contingent-unimodal group $(60 \%)$ and a significantly greater number $(45 \%)$ of the chicks in the contingentbimodal group demonstrated a PTD greater than $90 \%$, a significant difference between 
these groups was not seen. Prior studies have shown the merits of intersensory facilitation in affect discrimination, rhythm and tempo discrimination, numerical discrimination, word comprehension and segmentation, and abstract rule learning in the postnatal period. However, it seems that intersensory facilitation was not beneficial in the postnatal period in helping chicks remember information that was presented much earlier in development. It is not clear if we can conclude that intersensory facilitation does not help in the domain of memory. The bimodal redundant stimuli that were presented included an ecologically valid maternal call synchronized with a nonecologically valid patterned pulsing light. It is possible that the patterned pulsing light that was used along with the maternal call is not an appropriate stimulus to be used to answer our current question. The patterned pulsing light was used in this study because prior studies exploring the effects of intersensory redundancy prenatally have used this visual stimulus to stimulate bobwhite embryos, and upon hatching these chicks have shown to benefit from the audiovisual redundancy they experienced prior to hatching. It is possible that visual information is better received in the sequestered prenatal environment, but not in the early postnatal environment.

The current study also evaluated how different types of stimulus presentations effect memory retention. Contingency learning was compared to passive learning, which involves repeated exposure to the stimuli for long durations. Even though the contingent groups showed a significant preference for the familiarized call, a main effect of stimulus presentation did not emerge when the contingent and passive groups were compared with each other or when the contingent and the non-contingent groups were compared with each other. The lack of significant difference between the contingent and the non- 
contingent groups makes it hard to conclude the effectiveness of the contingent training. Results from within-group preference tests showed that the non-contingent group chicks and the passive group chicks did not show a preference for the familiarized call, whereas the chicks in the contingent groups showed a significant preference.

A significant difference between the contingent-bimodal and the passive-bimodal groups was seen, demonstrating an interaction between the contingency paradigm and the bimodal stimulus type. The chicks in the contingent-bimodal condition were able to remember the information longer under conditions of the bimodal redundant stimulus type in a contingency training paradigm. In the current study, a variant of the maternal call was presented contingent on the chick's vocalization. A social interaction such as this requires the chick to actively participate in the learning process whereas a passive condition lacks that kind of required interaction. However, there was no significant difference between the contingent-unimodal and the passive-unimodal groups, which could mean that it is not sufficient that the training be contingent for the chicks to remember the maternal call. Apart from the training being contingent, it appears that the stimulus type has to be bimodal and redundant in nature for the chicks to attend to it and remember for long durations.

An important question in the study of perceptual development is what causes certain properties of stimulation to be salient and attended to and other properties to be ignored. The IRH proposes that the redundancy of amodal information across sensory modalities attracts an organism's attention. Moreover, a contingent paradigm also recruits attention by making the training interactive, thus helping the organism attend to the stimuli. For instance, when a mother interacts with an infant, this interaction involves 
intersensory redundancy along with social contingencies. The auditory information she provides along with synchronized lip movements and facial expressions provide the intersensory redundancy. Moreover the dyadic interaction between the mother and infant usually involves a contingent relationship. Together, these may be necessary variables that help a young organism remember information during early development.

Even though the passive exposure was provided for 240 minutes (10 minutes/ hour) over a total duration of 24 hours, compared to the 5-minute contingent exposure, chicks from the passive groups did not show a preference for the familiar call in either the unimodal or bimodal conditions. Campbell and Jaynes (1966) found that periodic reinstatements, including partial repetitions of the initial experience, could maintain early memories into later developmental periods. On the basis of these findings it seemed plausible that repetitive training for long durations could also strengthen the memory trace. However the current study shows that this is not necessarily the case when the training style is passive. Even though previous studies have shown that redundant bimodal passive training in the prenatal period enhances memory for the familiarized maternal call, it is possible that the complex postnatal environment, with its varied perceptual and social experiences, presents a challenging environment for longer memory retention, compared to the sequestered prenatal environment of the egg (see also Lickliter \& Hellwell, 1992). Future studies could provide passive bimodal training to chicks in isolation to determine if the complex postnatal environment is a source of interference.

In summary, the current study showed that chicks remembered a familiarized maternal call for as long as 48 hours following exposure when they were trained contingently. Further, the contingent-bimodal group differed significantly from the 
passive-bimodal group, in that the contingent-bimodal group showed a significant preference for the familiarized call, whereas the passive-bimodal group did not. However there was not a significant main effect for contingency training, in spite of the high PTDs within the contingent groups. The large variability within the groups may be responsible for the lack of a main effect for contingency training, which does not allow for a clear distinction between the groups. Another reason for not finding significant difference between groups could be task difficulty. It is possible that asking 72 hour old chicks to remember a call presented two days earlier (one-third of their life so far) is too difficult a task, resulting in the large variability observed in the data. It is possible that making the task less challenging would reveal a distinction between the two exposure groups. For example, it may be useful to provide a 10-minute reminder to all the groups 48 hours after training and then test chicks at 96 hours of age. Further, it would also be worth investigating the effects of repeating contingency training for two consecutive days and then testing after a delay of 48 hours. If making the task less challenging results in a clear separation between the groups, the effects of the stimulus type and stimulus presentation can be better understood. 


\section{REFERENCES}

Bahrick,L.E., Gogate, L.J., and Ruiz, I. (2002). Attention and memory for faces and actions in infancy: The salience of actions over faces in dynamic events. Child Development, 73, 1629-1643.

Bahrick, L.E., \& Lickliter, R. (2000). Intersensory redundancy guides attentional selectivity and perceptual learning in infancy. Developmental Psychobiology, 36, 190-201.

Bahrick , L.E., \& Lickliter, R. (2002). Intersensory redundancy guides early perceptual and cognitive development. In R. Kail (Ed.), Advances in child development and behavior, Vol. 30, 153-187. New York: Academic Press.

Bahrick, L.E., Lickliter, R., Castellanos, I., \& Vaillant-Molina, M. (2010). Intersensory redundancy and tempo discrimination in infancy: The role of task difficulty and expertise. Developmental Science, 13, 731-737.

Bahrick, L. E., \& Pickens, J. N. (1994). Amodal relations: The basis for intermodal perception and learning. In D. Lewkowicz \& R. Lickliter (Eds.). The development of intersensory perception: Comparative perspectives, (pp. 205-233). Hillsdale, NI: Lawrence Erlbaum Associates.

Bahrick, L.E., \& Pickens, J.N. (1995). Infant memory for object motion across a period of three months: Implications for a four-phase attention function. Journal of Experimental Child Psychology, 59, 343-371.

Beecher, M. D \& Burt, J. M. (2004). The role of social interaction in bird song learning. Current Directions in Psychological Science, 13: 224.

Bauer, P. J. (1995). Recalling past events: From infancy to early childhood. Annals of Child Development, 11, 25-71.

Bauer, P. J. (1997). The development of memory in early childhood. In N. Cowan (Ed.), The development of memory in children, (pp. 83-111). Hove, England: Psychology Press.

Boller, K., Grabelle, M., \& Rovee-Collier, C. (1995). Effects of postevent information on infant's memory for a central target. Journal of Experimental Child Psychology, 59, 372-396.

Campbell, B. A., \& Jaynes, J. (1966). Reinstatement. Psychological Review, 55, 1-8.

Courage, M.L., \& Howe, M. L. (2004) Advances in early memory development research: Insights about the dark side of the moon, Developmental Review, 24, 6-32 . 
Flom, R., \& Bahrick, L. E. (2007). The development of infant discrimination of affect in multimodal and unimodal stimulation: The role of intersensory redundancy. Developmental Psychology, 43, 238-252.

Foushee, R., \& Lickliter, R. (2002). Early visual experience affects postnatal auditory responsiveness in bobwhite quail. Journal of Comparative Psychology, 116, 369380.

Flom, R. \& Bahrick, L. E. (2010). The effects of intersensory redundancy on attention and memory: Infants' long-term memory for orientation in audiovisual events. Developmental Psychology, 46, 428-436.

Frank, M.C., Slemmer, J., Marcus, G., \& Johnson, S. P. (2009). Information from multiple modalities helps 5-month-olds learn abstract rules. Developmental Science, 12, 504-509.

Gibson, E. J. and Pick, A. D. (2000). An ecological approach to perceptual learning and development. Oxford University Press, USA.

Galluccio, L., \& Rovee-Collier, C. (1999). Reinstatement effects on retention at 3 months of age. Learning and Motivation, 30, 296-316.

Gogate, L. J., \& Bahrick, L. E., (2001). Intersensory redundancy and seven-month-old infant's memory for arbitrary syllable-object relations. Infancy, 2, 219-231.

Gottlieb, G. (1993). Social induction of malleability in ducklings: Sensory basis and psychological mechanism. Animal Behaviour, 45, 707-719.

Harshaw, C., \& Lickliter, R. (2007). Interactive and vicarious acquisition of auditory preferences in Northern bobwhite (Colinus virginianus) chicks. Journal of Comparative Psychology, 121, 320-331.

Harshaw, C., Tourgeman, I. P., \& Lickliter, R. (2008). Stimulus contingency and the malleability of species-typical auditory preferences in northern bobwhite hatchlings. Developmental Psychobiology, 50, 460-472.

Hayne, H., Gross, J., Hildreth, K., \& Rovee-Collier, C. (2000). Repeated reminders increase the speed of memory retrieval by 3-month-old infants. Developmental Science, 3, 312-318.

Hollich, G., Newman, R.S., \& Jusczyk, P.W. (2005). Infant's use of synchronized visual information to separate streams of speech. Child Development, 76, 598-613. 
Honeycutt, H., \& Lickliter, R. (2002). Prenatal experience and postnatal perceptual preferences: Evidence for attentional-bias in bobwhite quail embryos. Journal of Comparative Psychology, 116, 270-276.

Jordon, K. E., Suanda, S. H., \& Brannon, E. M. (2008). Intersensory redundancy accelerates preverbal numerical competence. Cognition, 108, 210-221.

Lewkowicz, D. J., \& Lickliter, R. (Eds). (1994). Development of intersensory perception: Comparative perspectives. Hillsdale, NJ: Erlbaum.

Lickliter, R., Bahrick, L.E., \& Honeycutt, H. (2002). Intersensory redundancy facilitates prenatal perceptual learning in bobwhite quail (Colinus virginianus) embryos. Developmental Psychology, 38, 15-23.

Lickliter, R., Bahrick, L.E., \& Honeycutt, H. (2004). Intersensory redundancy enhances memory in bobwhite quail embryos. Infancy, 5, 253-269.

Lickliter, R., \& Hellewell, T. (1992). Contextual determinants of auditory learning in bobwhite quail embryos and hatchlings. Developmental Psychobiology, 25, 1731 .

Meltzoff, A. N., \& Kuhl, P. K. (1994). Faces and speech: Intermodal processing of biologically relevant signals in infants and adults. In D. J. Lewkowitz \& R. Lickliter (Eds.), The development of intersensory perception: Comparative perspectives. Hillsdale, NJ: Lawrence Erlbaum Assoc.

Ohr, P. S., Fagen, J. W., Rovee-Collier, C., Hayne, H., \& Linde, E. V., (1989). Amount of training and retention by infants. Developmental Psychobiology, 22, 69-80.

Rovee-Collier, C. (1995). Time windows in cognitive development. Developmental Psychology, 31, 147-169.

Rovee-Collier, C. (1996). Shifting the focus from what to why. Infant Behavior and Development, 19, 385-400.

Rovee-Collier, C., Hartshorn, K., \& DiRubbo, M. (1999). Long-term maintenance of infant memory. Developmental Psychobiology, 35, 91-102.

Rovee-Collier, C., \& Hayne, H. (1987). Reactivation of infant memory: Implications for cognitive development. In H. W. Reese (Ed.), Advances in child development and behavior, Vol. 20, 185-238. New York: Academic.

Rovee-Collier, C., Schechter, A., Shyi, G. C., \& Shields, P. J. (1992). Perceptual identification of contextual attributes and infant memory retrieval.

Developmental Psychology, 28, 307-318. 
Schulenberg, C. J., Riccio, D. C., \& Stikes, E. R. (1971) Acquisition and retention of a passive-avoidance response as a function of age in rats. Journal of Comparative and Physiological Psychology, 74, 75-83.

Spear, N. E. (1973). Retrieval of memory in animals. Psychological Review, 80, 163-194.

Spear, N. E., \& Kucharski, D. (1984). Ontogenetic differences in stimulus selection during conditioning. In R. V. Kail, \& N. E. Spear (Eds.), Comparative perspectives on the development of memory. Hillsdale, N.J.: Lawrence Erlbaum Associates.

Vaillant, J., Bahrick, L. E., \& Lickliter, R (2009). Detection of modality specific stimulus properties are enhanced by unimodal exposure during prenatal development. Poster presented at the Society for Research in Child Development meeting, Denver, CO 